\title{
Oculomotor Control, Brain Potentials, and Timelines of Word Recognition During Natural Reading
}

\author{
Reinhold Klieg ${ }^{* 1}$, Michael Dambacher ${ }^{*}$ Olaf Dimigen ${ }^{\ddagger}$ and Werner Sommer ${ }^{\ddagger}$ \\ *University of Potsdam, Germany \\ -University of Konstanz, Germany \\ ${ }^{\ddagger}$ Humboldt University, Berlin, Germany
}

\section{Introduction}

Reading is an outstanding achievement of the human brain. The ability to read has substantially formed our history and culture and plays an essential role in our everyday lives. Skilled readers can hardly prevent processing a written stimulus and, most often, they grasp a word's meaning within a fraction of a second. The understanding of the underlying mechanisms as well as the time course of this seemingly effortless skill is the goal of psycholinguistic research.

In general, two major sources of information contribute to language comprehension. First, bottom-up processes transmit neural codes of sensory input to increasingly complex levels. Upon success, the appropriate word representation in long-term memory is activated and semantic information associated with a word becomes available. Second, language-related knowledge and expectancies are expressed in top-down influences that guide the way words are understood. They permit the integration of word meaning into a wider context and hold the potential to bias expectations about upcoming words.

This is a preprint manuscript of the book chapter: Kliegl, R., Dambacher, M., Dimigen, O., \& Sommer, W. (2014). Oculomotor Control, Brain Potentials, and Timelines of Word Recognition during Natural Reading. In: Current Trends in Eye Tracking Research. (Editors: Horsley, M.; Eliot, M.; Knight, B.; \& Reilly, R.). Springer, pp. 141-155, DOI: 10.1007/978-3-319-02868-2_10. The final publication is available at:

http://link.springer.com/chapter/10.1007\%2F978-3-319-02868-2 10

\footnotetext{
${ }^{1}$ Please address correspondence to: kliegl@uni-potsdam.de
} 
Despite ample evidence for the relevance of bottom-up and top-down processes, their joint role in the timeline of word recognition is insufficiently understood. Clearly, bottom-up processes account for the elaboration of sensory signals and therefore reflect operations giving rise to the retrieval of a word's mental representation, i.e., lexical access. The role of top-down processes, however, is ambiguous. They may be slow and only play a role for mental operations after lexical access; alternatively, they may rapidly impinge on early lexical processes and co-determine the course of word identification.

As illustrated in Figure 1, when reading the words "In 365 days the earth circles the" the word "sun" will be predicted on the basis of our top-down knowledge. At the same time, when the eyes have landed on the second "the" of this sentence, the perceptual span (i.e., the area from which we process information "bottom-up"; McConkie and Rayner 1975; Rayner 1975) will include the word "sun." Disagreements about bottom-up and top-down processes in reading mainly relate to their dynamics, that is, about the timelines of their interaction and their localization within the brain. The coregistration of eye movements (EMs) and brain potentials is a promising research strategy to test theoretical proposals and resolve some of these disagreements.

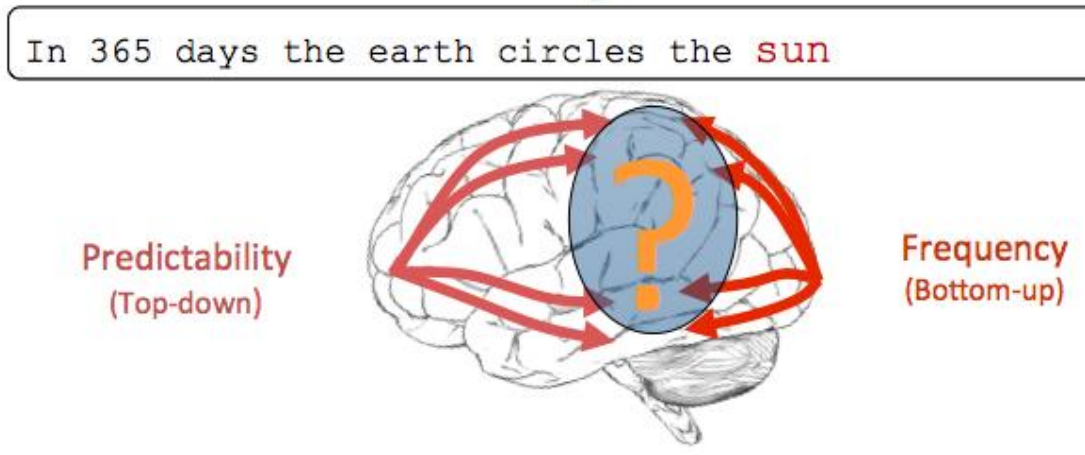

Figure 1. Illustration of top-down and bottom-up processing in reading: word predictability indexes top-down and word frequency for bottom-up processes

\section{Word Frequency and Predictability Affect Eye Movements and}

\section{Brain Potentials}

Two major determinants of the speed of word recognition can be considered as proxies of bottom-up and top-down streams in reading, respectively: word frequency and predictability. Normative word frequency is operationalized by counting the occurrences of a word in a large collection of documents. A quantitative index of word predictability is obtained with the cloze task. In this task, subjects are asked to guess the next word, given the previous words of a sentence (Taylor 1953) - the proportion 
of correct guesses serves as an index of predictability (cloze probability); that is, words are predicted from the prior context in the absence of the visual word form.
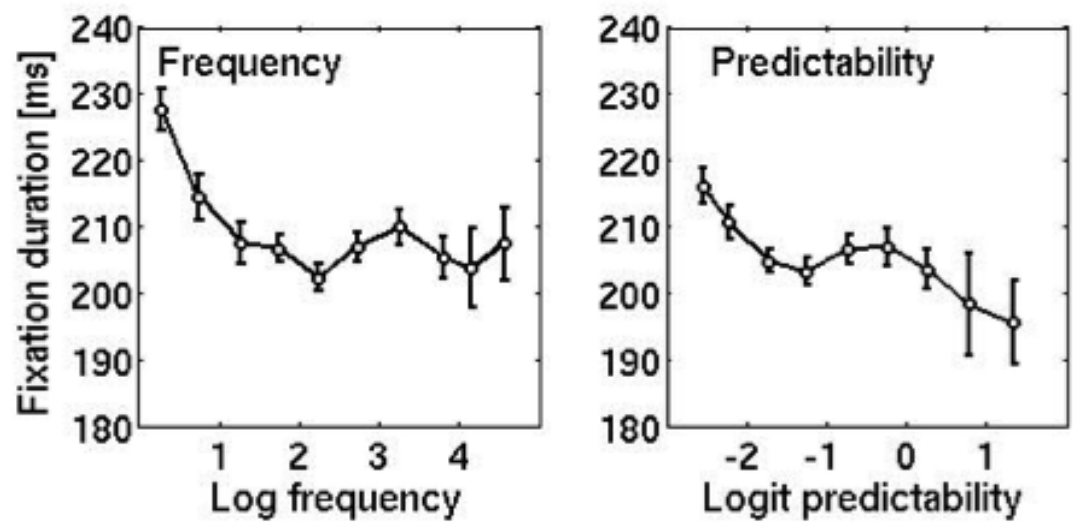

Figure 2. Frequency and predictability effects on single fixation durations (FDs) during reading of German sentences. (Kliegl et al. 2006)

\section{Evidence from Eye Movements (EMs)}

There is ample evidence that highly predictable words and highly frequent words require less processing time. Figure 2 illustrates these effects for single fixation durations (FDs) collected during the reading of German sentences (Kliegl et al. 2006; see also Ehrlich and Rayner 1981, Rayner and Duffy 1989). These effects relate to the fixated word. However, FDs on a given word are also influenced by the frequency and predictability of the neighboring words. Thus, FDs inform us about the preprocessing as well as postprocessing of words, when the eyes have already moved on to the next word. Such results are evidence for distributed processing of words across several fixations. These effects are typically small and are not found in every study (see exchange between Rayner et al. 2007; Klieg| 2007). In our opinion, the question is no longer whether or not such distributed processing occurs; the task is rather to determine which information is used under what conditions.

\section{Evidence from Event-Related Brain Potentials}

Frequency and predictability effects have also been demonstrated with event-related potentials (ERPs). One of the signature results of neurolinguistic research is the N400-component of the ERP, which is associated with the difficulty of retrieving stored word information (Kutas and Federmeier 2011; Kutas et al. 2006). In response to anomalous or unexpected words in a sentence (e.g., "The pizza was too hot to drink"), a pronounced negativity emerges about 200-250 ms after presentation of the 
anomalous target word (Kutas and Hillyard 1980), reaching a maximum at about $400 \mathrm{~ms}$. The N400component is also sensitive to variations in cloze probability as they occur while reading normal sentences without semantic violations (Dambacher et al. 2006; see right panel in Figure 3, based on the sentences used by Kliegl et al. 2006).

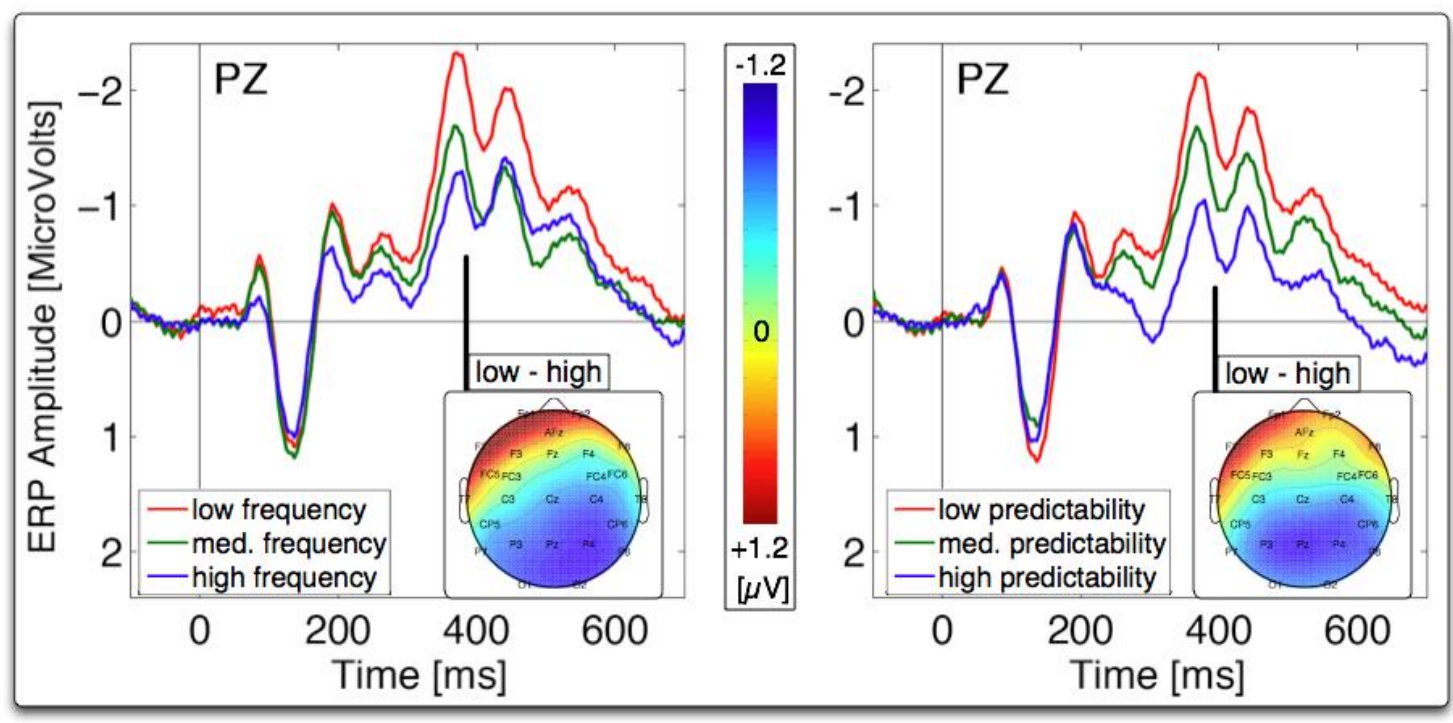

Figure 3. Frequency and predictability effects on N400-ERP amplitudes during reading German sentences. (Dambacher et al. 2006)

Dambacher et al. (2006) also reported larger N400 for low-frequency words compared to mediumand high-frequency words (Dambacher et al. 2006; left panel of Figure 3; see also Rugg 1990; Van Petten and Kutas 1990). Further, there is some evidence that frequency exerts influences on the ERP time course already before 200 ms (Hauk and Pulvermüller 2004; Sereno et al. 1998).

\section{Co-Registration of Eye Movements and Brain Potentials}

Natural Reading Versus Serial Visual Presentation. FDs are strongly affected not only by the properties of the currently fixated word but also by word frequency and predictability of the neighboring words (Kliegl et al. 2006). Dambacher and Kliegl (2007) assembled FDs measured on content words by Kliegl et al. (2006) during natural reading and ERPs measured on the same words by Dambacher et al. (2006) during the "traditional" ERP procedure, where words were presented serially at a fixed rate of $700 \mathrm{~ms}$ per word in the center of the display. Using path analysis based on words as units of observations, they were able to show that frequency and predictability of these content words mediated the covariation of FD and N400 amplitudes across successive words in sentences. 
Obviously, however, serial visual presentation (SVP) of words differs from natural reading in several important ways. First, fixation times are artificially inflated; a stimulus onset asynchrony (SOA) of 700 $\mathrm{ms}$ is about three times the duration of a normal fixation. Second, the SVP paradigm eliminates the need to select the next word target to which to saccade. Consequently, there is also no skipping of upcoming words or regressions to previous words in a sentence. Third, with central presentation of words in one location there is no preview of words to the right of fixation. Although some recent studies attempted to combine SVP with preview of the upcoming word, most SVP paradigms eliminate the core issue of much research on eye-movement control during reading: parafoveal preprocessing.

There are several reasons why most psycholinguistic ERP research uses the SVP paradigm rather than natural reading conditions. First, the SVP paradigm eliminates EMs. This is of paramount concern because EMs generate artifacts due to corneoretinal dipole changes and due to spike potentials (SPs) generated by extraocular muscles. These artifacts are often several magnitudes larger than the neurolinguistic experimental effects one hopes to identify with experimental manipulations of frequency or predictability. Second, the long SOAs reduce the temporal overlap with potentials triggered by the previous or the following word. The left column of Table 1 summarizes these problems. The table lists additional problems and solutions available or under active development. As far as these are not self-explanatory (e.g., the technical problems and their solutions), we refer readers to several recent expositions (e.g., Baccino 2011; Dimigen et al. 2009, 2011, 2012).

Despite the differences between natural and SVP reading, there is no doubt that ERP research has delivered important insights about timelines when various types of lexical and sublexical information become available (see, e.g., Kutas and Federmeier 2011; Lee et al. 2012, for reviews). Nevertheless, since we are interested in eye-movement control during reading with a focus on saccade target selection and parafoveal processing as well as in the timelines uncovered by ERP research, we need to tackle co-registration. Therefore, we adopted a two-pronged approach for our research program. On the one hand, we have started to co-register EMs and brain potentials during natural reading (Dimigen et al. 2011, 2012). On the other hand, we have also stayed with the SVP paradigm, but systematically varied SOA, using both slow presentation rates (SOA of 700 and $490 \mathrm{~ms}$ ) and a fast presentation rate (280 ms) that approximates the speed of natural reading (Dambacher et al. 2012). The general goal of this concerted effort addresses the important question of how timelines uncovered by traditional ERP research depend on SOA and whether there are task-specific dependencies that need to be taken into account.

Table 1. Methodological problems of co-recording of eye movements and EEG (Dimigen et al., 2011) 


\begin{tabular}{|c|c|}
\hline Problem & Solutions \\
\hline \multicolumn{2}{|l|}{ Technical } \\
\hline Data synchronization & Shared trigger pulses or D/A card \\
\hline $50-\mathrm{Hz}$ line noise & Notch filter \\
\hline Artifacts from head stabilization & Foam cushions or remote eye tracking \\
\hline \multicolumn{2}{|l|}{ Eye-movement artifacts } \\
\hline Corneoretinal dipole changes & Multiple source eye correction (MSEC) \\
\hline \multirow[t]{2}{*}{ Extraocular muscles (spike potential) } & $\begin{array}{l}\text { Independent component analysis, with component selection } \\
\text { supported by eye-tracking data }\end{array}$ \\
\hline & Constrain analysis to one fixation duration \\
\hline \multicolumn{2}{|l|}{ Overlapping signals } \\
\hline Temporal overlap with "neighbor" potentials & (Post hoc) matching of similar fixations \\
\hline \multirow[t]{2}{*}{ No neutral baseline } & Deconvolution methods \\
\hline & Modeling \\
\hline \multicolumn{2}{|l|}{ Low-level neural response variation } \\
\hline Saccade amplitude & (Post hoc) matching of similar fixations \\
\hline Luminance around fixated spot & Modeling, e.g., with linear mixed models \\
\hline
\end{tabular}

Fixation-Related Potentials During Natural Reading. The first step was to demonstrate that EEG recordings are feasible during natural reading; in other words, the problems listed in Table 1 are indeed surmountable. Using again the Potsdam sentences, which already featured in Figs. 2 and 3 , Dimigen et al. (2011) reported a fairly comprehensive experiment and data analysis. Using the multiple source eye correction method (MSEC; Berg and Scherg 1994; Ille et al. 2002), they were able to remove most of the corneoretinal artifacts. MSEC was only partially successful with the muscle SPS at the saccade onset (Keren et al. 2010). However, due to some topographic overlap with the corneoretinal dipole artifact, the MSEC procedure attenuated the contribution of the SP.

Fixation-Related Potentials. In traditional EEG research, the onsets of stimuli serve as the reference events at which EEG segments are aligned and averaged across trials, yielding the ERP. Analogous to this procedure, during natural reading, the onsets of fixations are used as synchronizing events. We refer to the averaged electrical potential that results from aligning EEG epochs with reference to the onsets of critical fixations as fixation-related potential (FRP). In addition, one can also calculate averaged potentials synchronized to the onsets of saccades (saccade-related potentials, SRPs). 

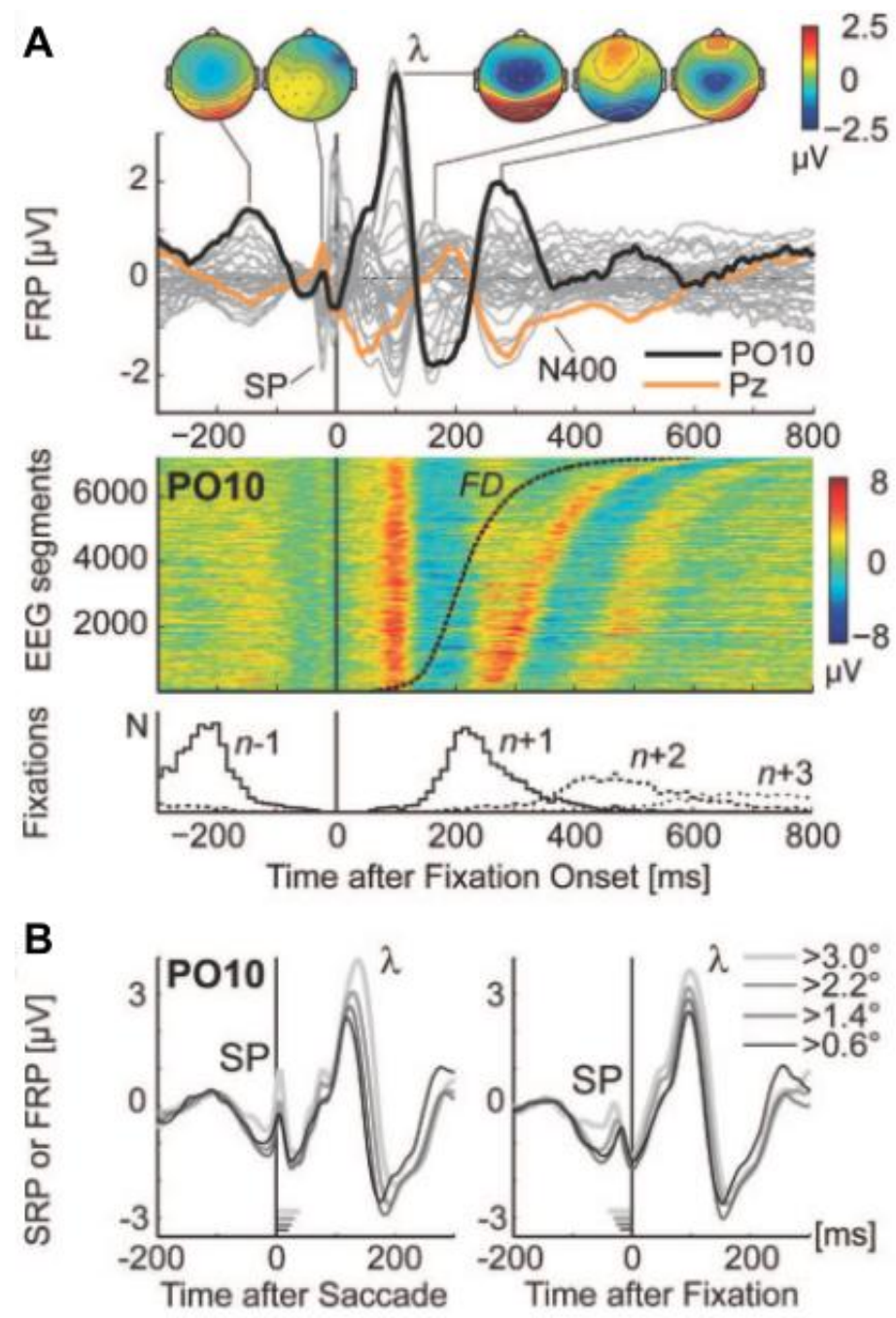

Figure 4. The fixation-related potential (FRP): illustrations of postsaccadic neural response (for explanation, see text). (Dimigen et al. 2011)

Deflections of the FRP. The maps at the top of Figure 4a illustrate scalp topographies of five interpretable FRP deflections: First, the visually evoked lambda response to the previous fixation N-1 occurs at around 150 ms prior to the current fixation N. Second, the muscular SP emerges at saccade onset. Third, the lambda response evoked by current fixation $\mathrm{N}$ peaks after around $100 \mathrm{~ms}$ at parietooccipital electrodes. Fourth, we observed an equivalent of the N170 component known from ERP research. Fifth, the $\mathrm{N} 400$ component overlapped with the lambda response to the next fixation $\mathrm{N}+1$. In general, the visually evoked FRP potentials show the P1-N1 complex known from ERP research. Before ocular artifact correction, many of the EEG channels were strongly correlated with the participants horizontal gaze position, as measured with the electrically independent eye tracker. After MSEC correction, correlations were nonsignificant or very small. In this way, simultaneous eye tracking can be used as an objective external criterion to evaluate the success of artifact correction. 
Neural Responses and FDs. In the lower panel of Figure 4a each of the 7,113 EEG segments contributing to the average FRP shown on the top is represented by one horizontal line. The segments are sorted by FD in increasing order. Colors represent EEG amplitudes at every sampling point that were smoothed vertically with a moving average across 50 adjacent segments. The figure shows that the positive peak at around $\mathbf{2 8 0} \mathrm{ms}$ in the average FRP is partially affected by the lambda response to fixation $\mathrm{N}+1$. The bottom panel of Figure 4a displays the distributions of onset latencies of the preceding and succeeding fixations relative to the onset of the current fixation $\mathrm{N}$.

SRPs and Saccade Amplitudes. Finally, Figure 4b shows that the morphology of SRPs and FRPs depend on saccade amplitude: Deflections of both the premotor SP and the visually evoked lambda response increased with saccade size. Because the lambda response largely dominates the shape of FRPs during left-to-right reading, saccade amplitudes can critically affect FRPs even after the correction of EM artifacts.

\section{The N400 Predictability Effect in FRPs During Natural Reading}

The N400 enjoys the status of a benchmark result in psycholinguistic research. Therefore, the first goal of a new methodology, such as the co-registration of EMs during reading, must be to replicate this signature result. As shown in Figure 5, this goal was indeed reached (see also Marton et al. 1985). The figure displays scalp distributions for differences between low- and high-predictability words for successive 40-ms windows after fixation onset. The large map at the right displays the aggregate for the usual N400 range. After correction for multiple testing, results were significant from $248 \mathrm{~ms}$ onwards, which is quite consistent with reports of N400 predictability effects in earlier SVP experiments (Kutas et al. 2006). An inspection of the maps, however, clearly suggests that the effect (a central negativity) emerges already somewhat earlier.

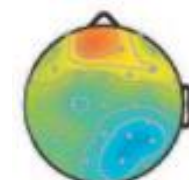

000-040

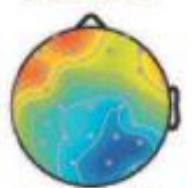

200-240
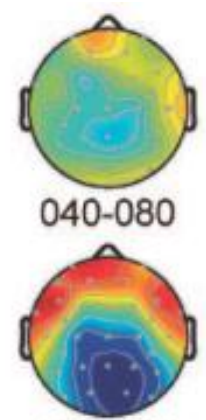

$240-280$
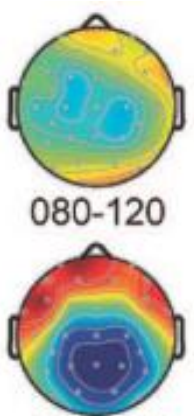

280-320
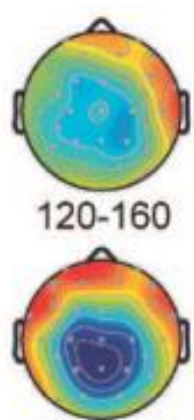

320-360

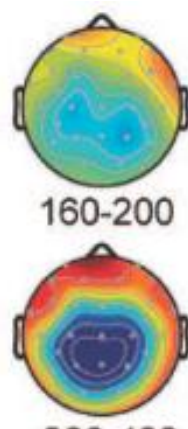

$360-400$

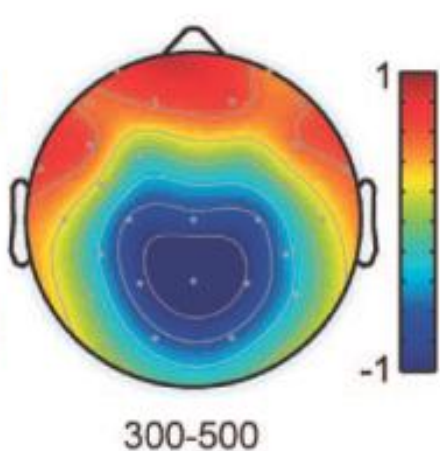

300-500

Figure 5. N400 effects of predictability (low minus high) in natural reading start early in fixation-related potentials (FRPs). (Dimigen et al. 2011) 


\section{Experimental Manipulation of Frequency and Predictability}

With the demonstration of a key result of psycholinguistic research, that is, the N400 predictability effect on FRPs, we are in a position to address the question whether the timelines of the effect are different in SVP studies. Thus, we are moving back to ERP research, but with the difference that we are looking at ERP effects at different SOAs (Dambacher et al. 2012). The motivation to examine SOA effects is straightforward: Traditional SVP experiments present one word at a time with relatively long intervals (usually from 500 up to 1000 ms) between successive stimuli. Natural reading, in contrast, takes place at a much faster pace of around 250 ms per word. The SOA manipulation therefore tests whether the rate of incoming words is a relevant factor for the time course of word recognition.

Moreover, because frequency and predictability are positively correlated in everyday language (cf., Dambacher and Kliegl 2007), we also aimed for uncorrelated effects of predictability and frequency in this research. This required the construction of new experimental material, in which high- and lowfrequency target words were embedded in identical neutral sentence frames. Predictability of these targets was manipulated by a preceding context sentence that induced high expectancy either of a high- or a low-frequency target, while its counterpiece was of low predictability (see Figure 6 for an illustration).

\section{Context sentence}

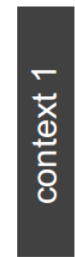

Der Mann auf dem Bild hantierte mit Modellen der Flotte des Columbus.

[The man on the picture fiddled around with models of Columbus' fleet.]

Der Mann auf dem Bild trug eine goldene Krone und $s a ß$ würdevoll auf einem Thron.

[The man on the picture wore a golden crown and sat stately on a throne.]
Neutral sentence

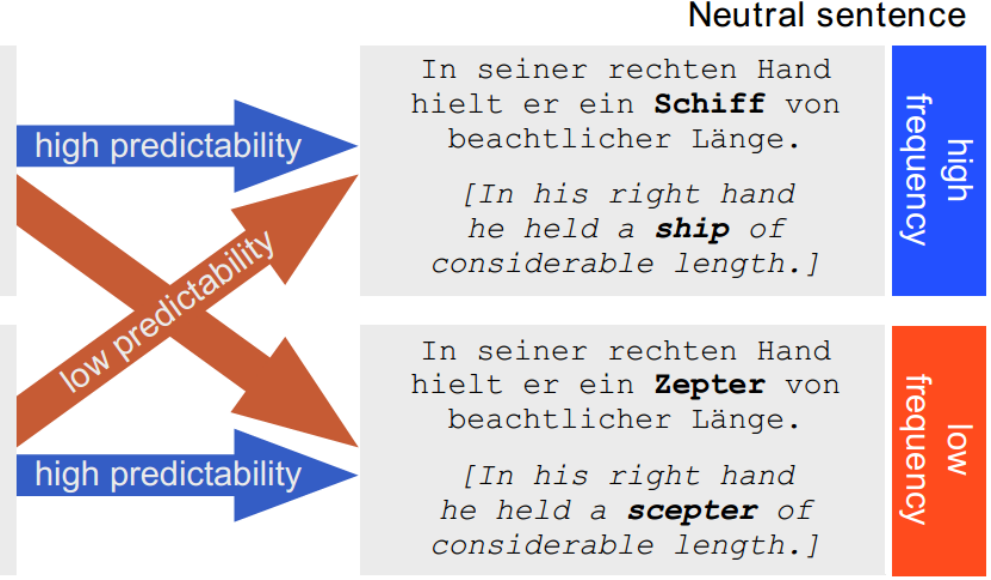

Figure 6. Experimental paradigm for orthogonal manipulation of frequency and predictability with target words. A set of low- and high-frequency words is presented in high- and low-predictability contexts. (Dambacher et al. 2009)

With this corpus, we conducted three SVP experiments with different presentation rates: SOAs of 700 and $490 \mathrm{~ms}$ reflected the medium and lower range of presentation times usually used in ERP experiments. The third SOA of 280 ms approximated normal reading speed. In another line of ongoing experiments, this corpus is also used to assess FRPs during normal left-to-right reading. 


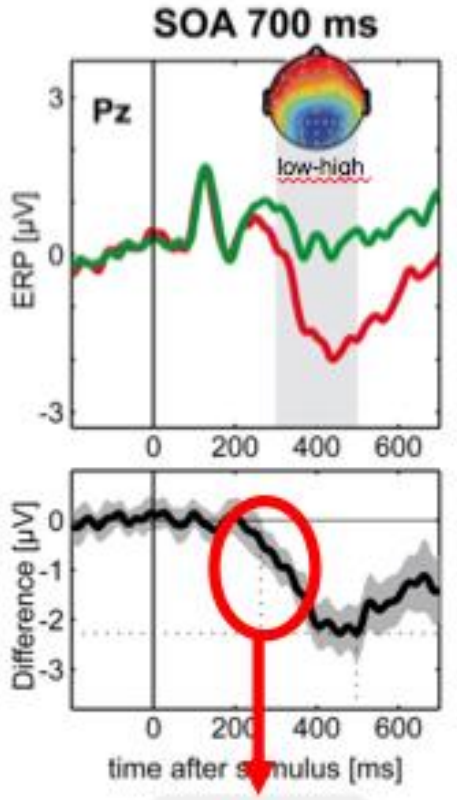

$263 \mathrm{~ms}$
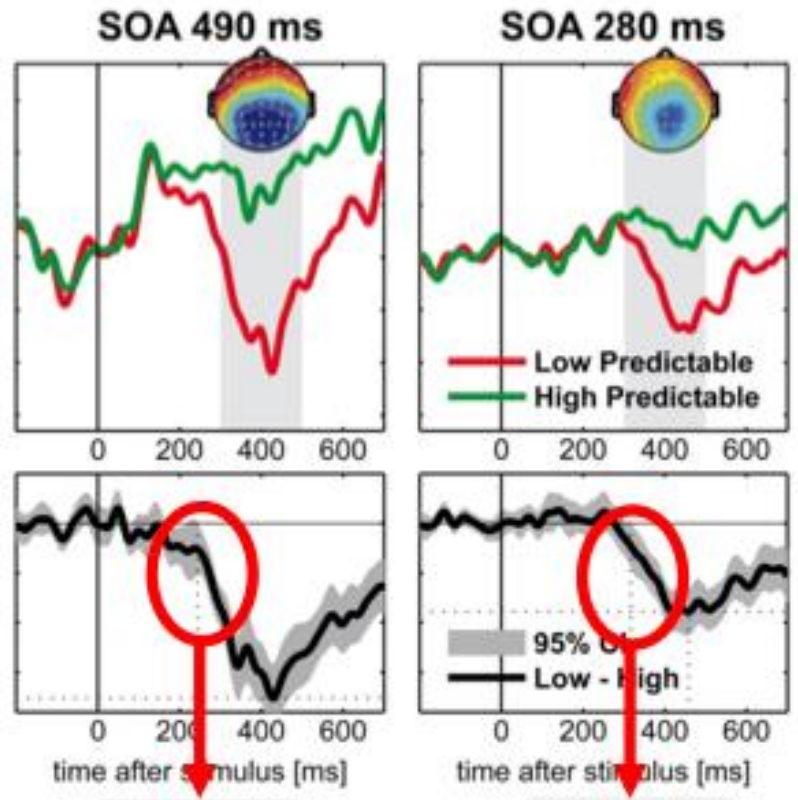

$263 \mathrm{~ms}$

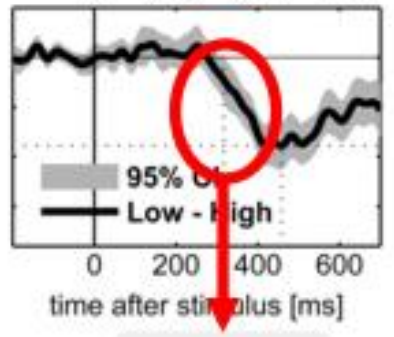

$313 \mathrm{~ms}$

Figure 7. Onsets of N400 predictability effect for (rapid) serial visual presentations (SVPs) with stimulus onset asynchronies (SOAs) of 700, 490, and $280 \mathrm{~ms}$. (Dambacher et al. 2012)

\section{Experimental Manipulation of SOA in the SVP Paradigm}

\subsection{The ERP Time Course Depends on SOA}

The first three experiments were devoted to documenting the SOA dependencies of predictability and frequency effects (Dambacher et al. 2012). SOA effects on the timeline of word recognition are not well established because fast SOAs have been rarely used in psycholinguistic ERP research. The reason is that overlapping components of successive words usually complicate the interpretation of neural responses since they cannot be attributed to individual words. Here, this problem was minimized since the words preceding and succeeding the target are physically identical across all conditions and only differ in the expectancy induced by the context sentence.

Predictability Effects. Figure 7 illustrates that SOA affected the N400 predictability effect. Its onset was later and amplitudes were smaller in the SOA of $280 \mathrm{~ms}$ compared to the SOAs of 490 and $700 \mathrm{~ms}$. One possible reason for the weaker N400 effect under a reading-like SOA is that the N400 temporally overlapped with the processing of the next word. Thus, two words may have been processed in parallel such that N400-related mechanisms were attenuated and/or decelerated. In fact, the offline 
comparison of SVP-based N400 amplitudes and FDs from natural reading revealed a cross talk between the currently fixated word and ongoing processing of the previous word (Dambacher and Kliegl 2007). It is also possible, though, that the N400 effect was smaller because the short SOA granted less time to build up expectations about the upcoming word. This limited semantic preactivation may result in a smaller predictability effect (e.g., Federmeier and Kutas 1999; Lau et al. 2008).

At the same time, however, the data of the short SOA revealed evidence that predictability supported lexical processing well before the N400. First, we observed a very early main effect of predictability at around 90 ms post-stimulus, which was reliable even within high- and low-frequency words. We suggested that this effect may point to a rapid verification mechanism, i.e., the comparison of the incoming with the expected word (Dambacher et al. 2009). In fact, similar early expectancy effects have been reported with spoken language (Van Berkum et al. 2005). Second, an interaction of frequency and predictability at around $145 \mathrm{~ms}$ pointed to an early processing advantage of highly predictable words. Notably, there was no evidence for such early predictability effects with the slower SOAs of 490 and $700 \mathrm{~ms}$.

Frequency Effects. Irrespective of SOA, Dambacher et al. (2012) found no evidence for frequency effects in the N400 range. However, frequency effects at earlier intervals indicated that the time course of lexical processing is modulated by SOA. Specifically, frequency effects emerged at 207, 174, and $131 \mathrm{~ms}$ with SOAs of 700, 490, and $280 \mathrm{~ms}$, respectively. There is one qualification: At the shortest SOA, the effect was only observed for high-predictable words. These results suggest that lexical processing is accelerated as presentation rate increases. In particular, the shortest SOA rate seems to play a special role for word recognition since only here early lexical processing was supported by predictability. Thus, normal reading speed may indeed reflect the optimal rate of visual word processing since it combines several sources of information to grant rapid lexical access. We want to emphasize that we consistently find frequency effects with robust and highly comparable scalp distributions at the latest around 200 ms. Analyses of frequency effects during natural reading are currently ongoing.

N400 Predictability Effects on FRPs During Natural Reading. In experiment 4 of this series, we coregistered EEG during natural reading of these sentences. Our preliminary analysis reveals predictability effects with an N400-like topography beginning as early as 160 ms. Thus, counter to the delay at the shortest relative to longer SOAs, there appears to be a mechanism that supports early retrieval of semantic information of upcoming words in left-to-right reading. One plausible candidate is parafoveal preview of the not-yet-fixated word, which is maintained in natural reading but 
prevented in SVP settings. In fact, the preview benefit effect, i.e., the reduction of FDs on words that have been visible from the parafovea compared to words that were parafoveally inaccessible, is well established in EM research and the brain correlates of the preview benefit effect has been recently tracked with FRPs (Dimigen et al. 2012).
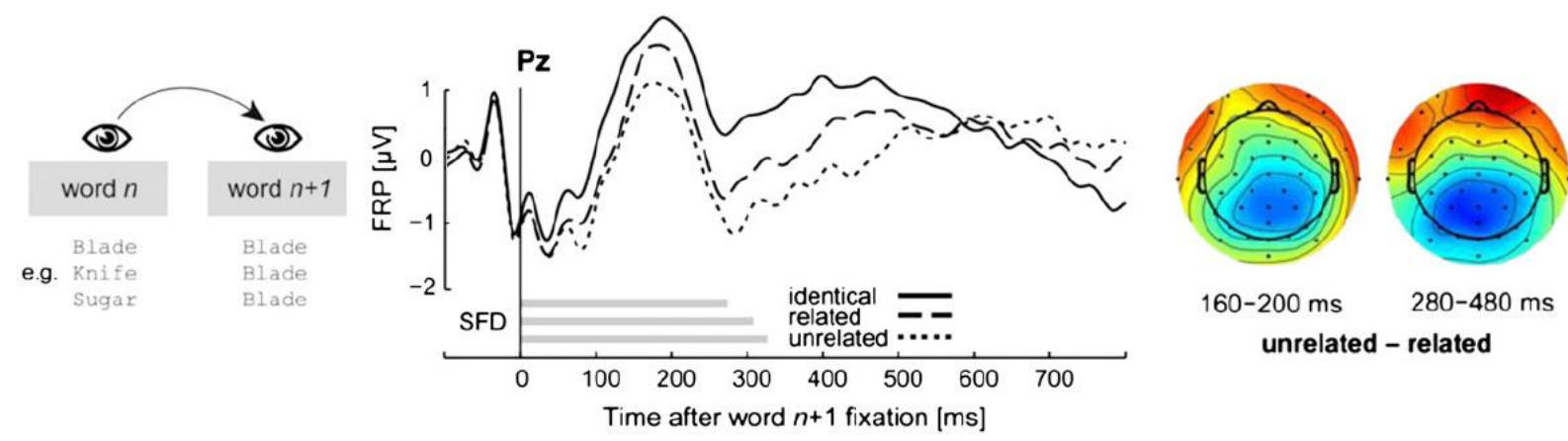

Figure 8. Priming effects on the N400 component while reading word lists. Within lists of five unrelated German nouns, two neighboring words were either semantically related (e.g., "knife"-"blade"), unrelated (e.g., "sugar""blade"), or identical (word repetition, e.g., "blade"-“blade"). The difference between fixating related versus unrelated word pairs modulated the N400 already 160 ms after fixating the second word (see the left scalp topography). The unusually early onset of semantic priming in fluent reading is most likely explained by the fact that readers had already preprocessed orthographical or phonological properties of the second word parafoveally. Figure adapted from Dimigen et al. (2012).

\section{Outlook: Gaze-Contingent Display Change Experiments}

The sentence material, comprising an orthogonal manipulation of frequency and predictability of content words, has already proved very useful to unravel SOA effects on timelines of predictability and frequency effects in ERPs during reading in the SVP procedure and, in ongoing research, in FRPs during natural reading. These results strongly suggest that readers make use of properties of words as soon as they are available in parafoveal vision in agreement with eye-movement research about parafoveal processing during reading. This research comprises multivariate analyses of the majority of words in sentences during natural reading (e.g., Kliegl et al. 2006) and the analyses of specific target words for which critical natural correlations have been eliminated by construction, such as the correlation between frequency and predictability (Dambacher et al. 2009; Figure 6).

In eye-movement research, statistical analyses, covering most words of sentences or a subset of target words, are complemented by experimental manipulations involving gaze-contingent display changes, such as the boundary paradigm (Rayner 1975). In the boundary paradigm, a target word (such as the target words of Figure 6) is either displayed all the time (like in natural reading) or its location is filled with a random string of letters and the word is displayed first during the saccade to the target word. 
Obviously, in this latter condition, preview of the target word is prevented just like in SVP. Typically, FDs are $\mathbf{2 0 - 5 0 ~ m s ~ l o n g e r ~ c o m p a r e d ~ t o ~ n a t u r a l ~ r e a d i n g . ~ T h i s ~ d i f f e r e n c e ~ i n ~ F D s ~ i s ~ c a l l e d ~ p r e v i e w ~ b e n e f i t . ~}$

Gaze-contingent display changes can also be employed during co-registration of EMs and EEG brain potentials (see Dimigen et al. 2012, for a first application and discussion of feasibility). Indeed, we already carried out two experiments to test predictability- and frequency-related preview effects for the target words used in the last set of experiments. In the first experiment, words were displayed during a saccade to the target word (i.e., the "classic" $N+1$-boundary paradigm); in the second experiment, words were displayed already when the eyes approached the word before the target word (i.e., the $\mathrm{N}+2$-boundary paradigm). Preliminary analyses suggested that preview effects were significant in FRPs for the $\mathrm{N}+1$-boundary condition; they were not significant for the $\mathrm{N}+2$-boundary paradigm. Specifically, if word $\mathrm{N}+1$ is masked, the $\mathrm{N} 400$ predictability effect was delayed-similar to the SOA 700 and SOA 490 timelines of Figure 7; if word $N+2$ was masked, the predictability occurred already after $160 \mathrm{~ms}$, that is, at the time when the effect appeared during natural reading. Thus, again, it appears that parafoveal preview exerts effects on timelines of word recognition during reading.

Clear evidence for the preview benefit in FDs and FRPs was also reported for reading a short list of five words with the goal to check for an animal name in the list (Dimigen et al. 2012). Like the sentence-reading studies, this study found that N400 effects are shifted forward during natural reading. As shown in Figure 8, effects of identity priming from showing repeated words in a list arose 80-120 ms after fixation onset. Similarly, semantic priming effects in the FRP started only $160 \mathrm{~ms}$ after fixation onset. Both latencies are significantly shorter than those typically observed for priming effects in SVP reading or in foveal priming studies that do not allow for parafoveal preview (e.g., Rugg 1987). This experiment also tested whether parafoveal preprocessing extends to semantic processing, but did not find any significant evidence in support of this hypothesis. Although semantic priming effects started early, they only began after a semantically related word was directly fixated. Similarly, in other trials of the experiment, which manipulated preview with the boundary paradigm, a semantically related preview word did not lead to shorter FDs or different brain responses than an unrelated preview word. These results stand in contrast to demonstrations of semantic relatedness with the boundary paradigm during reading of sentences (i.e., four experiments in Hohenstein and Kliegl 2012; see also Hohenstein et al. 2010, for evidence with a different, that is, parafoveal fast priming paradigm). Obviously, there are many empirical issues that remain to be sorted out, but we close with the observation that our research program on coregistration of EMs and brain potentials during reading closely recapitulated the development of eye-movement research on reading in a timecompressed manner. 


\section{Summary}

Our research on top-down predictability and bottom-up frequency effects on eye movements and brain potentials during reading covers experimental paradigms ranging from natural reading to SVP at different SOAs to gaze-contingent display changes. The experimental paradigms are needed to advance our theoretical understanding of the dynamics of word recognition during reading, but they also appear to incur paradigm-specific effects:

- Fixation-related EEG analyses in ecologically valid settings are feasible

- Care is necessary, since FRPs are influenced by a number of potentially confounding factors (e.g., artifacts, overlapping potentials, incoming saccade amplitude)

- N400 topography and amplitude are similar in natural reading and SVP, but there are large differences in timelines

- SOA manipulations in the SVP paradigm and gaze-contingent display-change manipulations (such as the boundary paradigm) show that this is (largely) explained by parafoveal preprocessing; this limits generalizability of ERP data recorded in the SVP paradigm for natural reading

Our understanding of eye-movement control during reading is shaped strongly by computational models of saccade generation during reading (e.g., Engbert and Kliegl 2011; Reichle 2011; Reilly and Radach 2009). Timelines of predictability and frequency effects and their interactions, as revealed by the co-registration of EMs and brain potentials, are important for the further development of these models, because they greatly reduce the degrees of freedom available for simulating this complex human activity. Most importantly, we present this research program as an example that it is possible to overcome this traditional fractionation of research domains in cognitive and biological psychology to the benefit, we hope, of everybody involved.

Acknowledgments. This research was supported by DFG Research Unit 868, Project A2. Address for correspondence: Reinhold Kliegl, Department of Psychology, University of Potsdam, Karl-Liebknecht-Str. 24/24, 14476 Potsdam, Germany. 


\section{References}

Baccino, T., \& Manunta, Y. (2005). Eye-fixation-related potentials: Insight into parafoveal processing. Journal of Psychophysiology, 19, 204-215.

Baccino, T. (2011). Eye movements and concurrent event-related potentials: Eye fixation-related potential investigations in reading. In: S.P. Liversedge, I.D. Gilchrist, \& S. Everling (Eds.), Eye Movements Handbook (pp. 857-870). Oxford: Oxford University Press.

Berg, P., \& Scherg, M. (1994). A multiple source approach to the correction of eye artifacts. Electroencephalography and Clinical Neurophysiology, 90, 229-241.

Dambacher, M. (2010). Bottom-up and top-down processes in reading. Potsdam: Universitätsverlag Potsdam.

Dambacher, M., Dimigen, O., Braun, M., Wille, K., Jacobs, A. M., \& Kliegl, R. (2012). Stimulus onset asynchrony and the timeline of word recognition: Event-related potentials during sentence reading. Neuropsychologia, 50(8), 1852-70

Dambacher, M., \& Kliegl, R. (2007). Synchronizing timelines: Relations between fixation durations and N400 amplitudes during sentence reading. Brain Research, 1155, 147-162.

Dambacher, M., Kliegl, R., Hofmann, M., \& Jacobs, A. M. (2006). Frequency and predictability effects on eventrelated potentials during reading. Brain Research, 1084, 89-103.

Dambacher, M., Rolfs, M., Göllner, K., Kliegl, R., \& Jacobs, A. M. (2009). Event-related potentials reveal rapid verification of predicted visual input. PLoS ONE, 4(3), e5047.

Dimigen, O., Kliegl, R., \& Sommer, W. (2012). Trans-saccadic parafoveal preview benefits in fluent reading: A study with fixation-related brain potentials. Neurolmage, 62, 381-393.

Dimigen, O., Sommer, W., Hohlfeld, A., Jacobs, A. M., \& Kliegl, R. (2011). Co-registration of eye movements and EEG in natural reading: Analyses and review. Journal of Experimental Psychology: General, 140, 552-572.

Dimigen, O., Valsecchi, M., Sommer, W., \& Kliegl, R. (2009). Human microsaccade-related visual brain responses. Journal of Neuroscience, 29, 12321-12331.

Engbert, R., \& Kliegl, R. (2011). Parallel graded attention models of reading. In: S.P. Liversedge, I.D. Gilchrist, \& S. Everling (Eds.), Eye Movements Handbook (pp. 787-800). Oxford: Oxford University Press.

Ehrlich, S. F., \& Rayner, K. (1981). Contextual effects on word recognition and eye movements during reading. Journal of Verbal Learning and Verbal Behavior, 20, 641-655.

Hauk, O., \& Pulvermüller, F. (2004). Effects of word length and frequency on the human event-related potential. Clinical Neurophysiology, 115, 1090-1103.

Hohenstein, S., \& Kliegl, R. (2012). Semantic preview benefit during reading. Manuscript submitted for publication. 
Hohenstein, S., Laubrock, J., \& Kliegl, R. (2010). Semantic preview benefit in eye movements during reading: A parafoveal fast-priming study. Journal of Experimental Psychology: Learning, Memory, and Cognition, 36, 1150-1170.

Ille, N., Berg, P., \& Scherg, M. (2002). Artifact correction of the ongoing EEG using spatial filters based on artifact and brain signal topographies. Journal of Clinical Neurophysiology, 19,

Keren, A. S., Yuval-Greenberg, S., \& Deouell, L. Y. (2010). Saccadic spike potentials in gammaband EEG: Characterization, detection, and suppression. Neurolmage, 49, 2248-2263.

Kliegl, R. (2007). Toward a perceptual-span theory of distributed processing in reading: A reply to Rayner, Pollatsek, Drieghe, Slattery, and Reichle (2007). Journal of Experimental Psychology: General, 136, 530-537.

Kliegl, R., Dambacher, M., Dimigen, O., Jacobs, A. M., \& Sommer, W. (2012). Eye movements and brain electric potentials during reading. Psychological Research, 76, 145-158.

Kliegl, R., Nuthmann, A., \& Engbert, R. (2006). Tracking the mind during reading: The influence of past, present, and future words on fixation durations. Journal of Experimental Psychology: General, 135, 12-35.

Kretzschmar, F., Bornkessel-Schlesewsky, I., \& Schlesewsky, M. (2009). Parafoveal versus foveal N400s dissociate spreading activation from contextual fit. Neuroreport, 20, 1613-1618.

Kuperman, V., Dambacher, M., Nuthmann, A., Kliegl, R., (2010). The effect of word position on eye-movements in sentence and paragraph reading. Quarterly Journal of Experimental Psychology, 63, 1838-1357.

Kutas, M., \& Federmeier, K. D. (2011). Thirty years and counting: Finding meaning in the N400 component of the event-related brain potential (ERP). Annual Review of Psychology, 62, 621-647.

Kutas, M., \& Hillyard, S. A. (1980). Reading senseless sentences: Brain potentials reflect semantic incongruity. Science, 207, 203-205.

Kutas, M., Van Petten, C., \& Kluender, R. (2006). Psycholinguistics electrified II: 1994-2005. In M. Gernsbacher \& M. Traxler (Eds.), Handbook of psycholinguistics, 2nd edition (pp. 659-724). New York: Elsevier Press.

Lee, C.-Y., Liu, Y.-N., \& Tsai, J.-L. (2012). The time course of contextual effects on visual word recognition. Frontiers in Psychology. doi:10.3389/fpsyg.2012.00285.

McConkie, G. W., \& Rayner, K. (1975). The span of effective stimulus during a fixation in reading. Perception \& Psychophysics, 17, 578-586.

Rayner, K. (1975). The perceptual span and peripheral cues in reading. Cognitive Psychology, 7, 65-81. Rayner, K., \& Duffy, S. (1986) Lexical complexity and fixation times in reading: Effects of word frequency, verb complexity, and lexical ambiguity. Memory \& Cognition, 14, 191-201.

Rayner, K., Pollatsek, A., Drieghe, D., Slattery, T., \& Reichle, E. D. (2007). Reichle, E. D. (2011). Serial-attention models in reading. In: S.P. Liversedge, I.D. Gilchrist, \& S. Everling (Eds.), Eye Movements Handbook (pp. 767786). Oxford: Oxford University Press. 
Reilly, R. G., \& Radach, R. (2006). Some empirical tests of an interactive activation model of eye movement control in reading. Journal of Cognitive Systems Research, 7, 34-55.

Rugg, M. D. (1987). Dissociation of semantic priming, word and non-word repetition effects by event-related potentials. Quarterly Journal of Experimental Psychology, 39, 123-148.

Rugg, M. D. (1990). Event-related brain potentials dissociate repetition effects of high- and low-frequency words. Memory and Cognition, 18, 367-379.

Taylor, W. L. (1953). Cloze procedure: A new tool for measuring readability. Journalism Quarterly, 30, 415-433.

Sereno, S. C., \& Rayner, K. (2003). Measuring word recognition in reading: Eye movements and event- related potentials. Trends in Cognitive Science, 7, 489-493.

Sereno, S. C., Rayner, K., \& Posner, M. I. (1998). Establishing a time-line of word recognition: Evidence from eye movements and event-related potentials. Neuroreport, 9, 2195-2200.

Van Berkum, J. J. A., Brown, C. M., Zwitserlood, P., Kooijman, V., \& Hagoort, P. (2005). Anticipating upcoming words in discourse: Evidence from ERPs and reading times. Journal of Experimental Psychology: Learning, Memory, and Cognition, 31, 443-467.

Van Petten, C., \& Kutas, M. (1990). Interactions between sentence context and word frequency in event-related brain potentials. Memory and Cognition, 18, 380-393. 